\title{
Shellfish Mix Antigen IgE Antibody Measurement
}

National Cancer Institute

\section{Source}

National Cancer Institute. Shellfish Mix Antigen IgE Antibody Measurement. NCI

Thesaurus. Code C130120.

A measurement of the shellfish mix antigen IgE antibody in a biological specimen. 